\title{
Estratégias de Saúde da Família: Análise dos Métodos Gerenciais dos Enfermeiros na Perspectiva do Processo Administrativo Neoclássico
}

\author{
Fernanda Mathioni ${ }^{1}$, Jacinta Spies ${ }^{2}$
}

\begin{abstract}
RESUMO
A pesquisa objetivou analisar o método de gestão adotado pelos enfermeiros, sob a luz das funções básicas da Administração. Seguiu-se uma abordagem quantitativa, exploratório- descritiva. O estudo de campo foi realizado nas Estratégias de Saúde da Família de três munícipios de Gestão Plena da Atenção Básica Ampliada, pertencentes a 14a Coordenadoria Regional de Saúde do Estado do Rio Grande do Sul. A coleta de dados ocorreu no período de fevereiro e março de 2017, por meio de um formulário aplicado aos entrevistados. $O$ tratamento dos dados foi feito por meio da análise estatística descritiva, utilizando-se o método de distribuição de frequências. Como principais resultados obteve-se que: $52,94 \%$ dos sujeitos pesquisados não possuem capacitação na área de gerenciamento; $76,47 \%$ relataram que as esferas do governo não têm demonstrado intenção de capacitá-los para gerir suas equipes; $64,71 \%$ sentem-se aptos para exercer sua função gerencial na ESF, porém $47,06 \%$ não seguem nenhuma metodologia específica para orientar sua gestão; 70,59\% não estão a par do planejamento estratégico de sua Secretaria Municipal de Saúde; $88,24 \%$ possuem controle das atividades realizadas em sua ESF por meio de relatórios, porém $80,00 \%$ não fazem a análise, com a equipe, dos dados presentes nos relatórios periodicamente. Em resumo, os dados colhidos indicam que nos municípios estudados o processo administrativo não está consolidado integralmente, há deficiências, principalmente no planejamento e controle de desempenho.
\end{abstract}

Palavras-chave: Estratégia Saúde da Família. Enfermagem. Gerenciamento.

\section{FAMILY HEALTH STRATEGIES: ANALYSIS OF MANAGEMENT METHODS OF NURSES} IN THE PERSPECTIVE OF THE NEOCLASSICAL ADMINISTRATIVE PROCESS

\begin{abstract}
The research aimed to analyze the management method adopted by Nurses, in light of the basic functions of administration. A quantitative, exploratory, descriptive approach was followed. The field study was carried out in the Family Health Strategies (FHS) of three cities of Full Management of Expanded Primary Care, belonging to the $14^{\text {th }}$ Regional Health Coordination of the state of Rio Grande do Sul. The data collection took place in the period of February and March of 2017, through a form applied to the interviewees. The data were processed through descriptive statistical analysis, using the frequency distribution method. As main results it was obtained that: $52,94 \%$ of the subjects surveyed do not have training in the area of management; $76,47 \%$ reported that government spheres have not demonstrated intent to empower them to manage their teams; $64,71 \%$ feel able to perform their managerial function in the FHS, but $47,06 \%$ does not follow any specific methodology to guide their management; $70,59 \%$ are not aware of the strategic planning of their Municipal Health Department; $88,24 \%$ have control of the activities carried out in their FHS through reports, but $80,00 \%$ do not analyze the data in the reports periodically with the team. In summary, the data collected indicate that in the cities studied the administrative process is not fully consolidated, there are deficiencies, mainly in planning and performance control.
\end{abstract}

Keywords: Family Health Strategy. Nursing. Management.

RECEBIDO EM: 23/9/2017

MODIFICAÇÕES REQUERIDAS EM: 1/7/2019

ACEITO EM: 11/10/2019

\footnotetext{
Graduada em Enfermagem pela Sociedade Educacional Três de Maio (2018). Cursando a Residência Multiprofissional Integrada em Saúde Coletiva na Universidade Federal do Rio Grande do Sul (2018-2020). fernandamathioni@hotmail.com

2 Graduada em Enfermagem e Obstetrícia - Licenciatura pela Faculdade de Enfermagem Nossa Senhora Medianeira (1981). Especialista em Administração Hospitalar pelo Centro Universitário São Camilo - Campus Pompeia (1989). Especialista em Gerência de Serviços de Enfermagem pela Universidade Regional do Noroeste do Estado do Rio Grande do Sul (2003). Atua como Coordenadora do Núcleo de Segurança do Paciente no Hospital Vida e Saúde da Associação Hospitalar Caridade de Santa Rosa. Professora da Graduação em Enfermagem e do Técnico em Enfermagem na Sociedade Educacional Três de Maio. jacintasetrem@yahoo.com.br
} 


\section{INTRODUÇÃO}

Desde 2010, por meio da Portaria no 4.279, o Sistema Único de Saúde (SUS) vem sendo reorganizado em Redes de Atenção à Saúde (RAS). A gestão nos âmbitos municipal, regional, estadual e nacional tem articulado os serviços de saúde em distintos níveis de densidade tecnológica e criado linhas de ação com outros setores vinculados aos fatores condicionantes da saúde (BRASIL, 2017).

A RAS é composta principalmente pelos pontos de atenção secundários e terciários, que desempenham suas atividades com tecnologias de média e alta densidade, como em hospitais e Unidades de Pronto Atendimento; e pelo ponto de atenção primária, responsável pelo trabalho de menor densidade tecnológica, porém de maior complexidade, atuando principalmente na prevenção de agravos e complicações, por meio das Estratégias de Saúde da Família (ESF) e equipes com outras configurações (ALBUQUERQUE, 2015).

"A Atenção Primária à Saúde (APS) caracteriza-se como a porta de entrada preferencial da RAS, acolhendo usuários e promovendo a vinculação e responsabilização pela atenção a suas necessidades de saúde" (POÇAS; FREITAS; DUARTE, 2017, p. 2). 0 modelo estrutural predominante na APS é a ESF. Esta é configurada pelo atendimento a uma área geográfica predeterminada abrangendo uma população de, aproximadamente, 4 mil e composta por uma equipe multiprofissional, formada por médico, cirurgião-dentista, agentes comunitários de saúde, técnicos ou auxiliares em enfermagem e enfermeiro, sendo este último o coordenador da equipe, conforme Ribeiro, Santos e Meira (2006) e Dias et al. (2012).

Nessa perspectiva, de que a atuação do enfermeiro nos sistemas de saúde, principalmente nas ESFs, baseia-se no processo de cuidar e administrar, Almeida (2014) observa que este profissional, para realizar um trabalho satisfatório e de qualidade, deve empregar instrumentos como o planejamento, a organização, a coordenação e o controle. Estas funções caracterizam um ciclo denominado Processo Administrativo Neoclássico, o qual possibilita constante correção e ajustes de ações e serviços dentro de um sistema (CHIAVENATO, 2001). Ademais, o gestor pode utilizar as ferramentas de qualidade para instrumentalizar sua gestão, as quais são técnicas que facilitam a mensuração, análise e criação de solução para problemas, auxiliando no bom desempenho dos processos de trabalho (ALVES, 2012).
Os avanços estruturais do SUS nas últimas décadas transformaram significativamente sua funcionalidade, propiciando um maior acesso aos serviços e, consequentemente, mais qualidade de vida aos usuários. A continuidade deste progresso está associada ao gerenciamento apropriado das atividades, especialmente na APS, que é o centro de comunicação da rede, e desta forma nos motiva a refletir sobre como tem-se dado o processo de coordenar as ESFs (BARBOSA, 2014).

Acredita-se que estudos dessa natureza possam auxiliar na melhoria contínua dos sistemas de saúde, na estruturação dos seus processos de trabalho e estabelecimento de organizações sadias, eficientes e eficazes em prol da população.

Reconhecendo a importância do vínculo da Enfermagem e os conhecimentos administrativos, e a APS como possível local de atuação da categoria, a presente pesquisa teve como objetivo analisar o método de gestão adotado pelos enfermeiros nas ESFs, a fim de verificar se suas ações estão baseadas na improvisação e empirismo ou estruturadas em um plano; se há organização; controle e direção do serviço.

\section{MATERIAIS E MÉTODOS}

Trata-se de uma pesquisa com abordagem quantitativa, exploratório-descritiva. O presente estudo foi encaminhado à Plataforma Brasil e aprovado pelo Comitê de Ética em Pesquisa (CEP) da Universidade de Passo Fundo, evidenciado pelo termo consubstanciado no 61857316.3.0000.5342.

A pesquisa foi realizada nas ESFs, de três munícipios de Gestão Plena da Atenção Básica Ampliada, pertencentes a 14a Coordenadoria Regional de Saúde (CRS) do Estado do Rio Grande do Sul (RS) e desenvolvida no decorrer do primeiro semestre de 2017.

A escolha dos municípios ocorreu por meio do mapeamento da região da 14a CRS/RS, com o auxílio do censo do Instituto Brasileiro de Geografia e Estatística (IBGE) de 2010 e informações das prefeituras, em que se buscou um perfil populacional, socioeconômico e estrutura de saúde semelhante. Nesse contexto, foram incluídos na pesquisa os municípios pertencentes à CRS já citada, com Gestão Plena da Atenção Básica Ampliada, uma população entre 10.000 e 25.000 habitantes, com Índices de Desenvolvimento Humano (IDH) semelhantes, e que dispõem de, pelo menos, cinco ESFs e, consequentemente, de cinco ou mais enfermeiros que atuam nestas Unidades de Saúde (US). Foram excluídos da pesquisa os municípios dessa re- 
gião estruturados como Gestão Plena do Sistema Municipal e que não se enquadraram nos demais critérios de inclusão citados anteriormente.

Conforme as informações levantadas, foram selecionados três municípios para a execução da pesquisa, apresentados no Quadro 1 por letras, mantendo-se $o$ anonimato.

Quadro 1 - Informação dos municípios escolhidos para a pesquisa

\begin{tabular}{|c|c|c|c|c|}
\hline Município & $\begin{array}{c}\text { No de } \\
\text { habitantes }\end{array}$ & IDH & $\begin{array}{c}\text { No de } \\
\text { ESFs }\end{array}$ & $\begin{array}{c}\text { No de } \\
\text { Enfermeiros }\end{array}$ \\
\hline A & 23.726 & 0,759 & 7 & 7 \\
\hline B & 18.348 & 0,783 & 6 & 6 \\
\hline C & 14.378 & 0,738 & 5 & 5 \\
\hline
\end{tabular}

Fonte: Adaptado com informações do IBGE (2010) e das prefeituras (2017).

Quanto aos sujeitos, foram incluídos, em caráter intencional, todos os enfermeiros que trabalhavam nessas ESFs, que aceitaram, de forma voluntária, participar da pesquisa e atuantes no momento da coleta de dados. Desta forma, 18 enfermeiros compõe a população da pesquisa, no entanto no período da coleta de dados uma profissional encontrava-se em licença-maternidade, não sendo portanto incluída, contabilizando uma amostra de 17 participantes.

A coleta de dados foi realizada por meio da aplicação de um formulário aos partícipes da pesquisa, no período de fevereiro e março de 2017, com questões abertas e fechadas.

Para a estruturação do banco de dados e sua posterior análise empregou-se o software Epi Info 7. 0 tratamento dos dados ocorreu mediante análise estatística descritiva, utilizando o método de distribuição de frequências. Os resultados foram expressos em tabelas na forma de frequência absoluta e percentual.

No decurso do estudo foram atendidas as normas presentes na Resolução no 466/2012 do Conselho Nacional da Saúde, que se ocupa da ética em pesquisas envolvendo seres humanos. Contemplando estes aspectos, foi solicitada, à 14a CRS/RS, uma autorização para a coleta de dados nos municípios escolhidos, assim como leitura e assinatura do Termo de Consentimento Livre e Esclarecido (TCLE) pelos enfermeiros participantes.

\section{RESULTADOS E DISCUSSÃO}

Participaram da pesquisa 16 mulheres $(94,12 \%)$ e 1 homem (5,88\%), demonstrando a predominância feminina na área da Enfermagem. Tal característica também é visível na pesquisa sobre o perfil dos profis- sionais da Enfermagem no país, realizada pelo ConseIho Federal de Enfermagem (Cofen) e a Fundação Instituto Oswaldo Cruz (Fiocruz) (MACHADO et al., 2016).

A idade dos participantes se distribuiu da seguinte forma: de 21 a 30 anos -5 sujeitos $(29,41 \%)$; de 31 a 40 anos -7 sujeitos (41,18\%); de 41 a 50 anos - 2 sujeitos (11,76\%); de 51 anos em diante -3 sujeitos (17,65\%), expressando a predominância de participantes entre 31 e 40 anos, seguido de 21 a 30 anos de idade, condizendo com a pesquisa do Cofen/Fiocruz, que revela que a Enfermagem é uma categoria jovem, em pleno rejuvenescimento, pois 1 milhão e 100 mil trabalhadores $(61,7 \%)$ do total dos seus sujeitos pesquisados têm até 40 anos de idade (MACHADO et al., 2016).

A escolaridade dos participantes distribuiu-se em 7 sujeitos $(41,18 \%)$ com Ensino Superior completo e 10 sujeitos (58,82\%) com Pós-Graduação. A maior parte dos profissionais que possuem somente o Ensino Superior completo encontra-se na faixa dos 21 a 31 anos $(42,86 \%)$ e, destes, $28,57 \%$ estão cursando uma Pós-Graduação, demonstrando, conforme Silva, Motta e Zeitoune (2010), o interesse da nova geração de profissionais em se especializar.

Quanto ao tempo de atuação como enfermeiros da Atenção Primária à Saúde tem-se: de 0 a 2 anos - 2 sujeitos (11,76\%); de 2 a 5 anos -4 sujeitos $(23,53 \%)$; e mais de 5 anos -11 sujeitos $(64,71 \%)$.

A seguir são apresentados os resultados envolvendo a coordenação diretamente.

Tabela 1 - Número de profissionais com especialização ou curso de capacitação em gestão

\begin{tabular}{ccc}
\hline Resposta & Frequência & Percentual \\
\hline Não & 9 & $52,94 \%$ \\
\hline Sim & 8 & $47,06 \%$ \\
\hline Total & 17 & $100 \%$ \\
\hline \multicolumn{3}{c}{ Fonte: Elaborada pelas autoras. }
\end{tabular}

A Tabela 1 mostra o número de entrevistados conforme seu aperfeiçoamento em gestão, sendo que a maioria $(52,94 \%)$ não possui nenhum curso de capacitação nesta área; e dos 8 sujeitos $(47,06 \%)$ que possuem, 37,5\% têm Especialização focada na gestão, e os demais $(62,5 \%)$ têm cursos de aperfeiçoamento com até 160 horas, oferecidos pelo Conselho Regional de Enfermagem (Coren) e Ministério da Saúde (MS), por intermédio da $14^{\circ}$ CRS em sua maioria.

Na pesquisa de Neto e Sampaio (2007) verificou-se a prevalência de enfermeiros como gestores das ESFs no município de Sobral - CE, e também, que $71,4 \%$ possuíam Especialização na modalidade de 
Residência Multiprofissional em Saúde da Família e, mesmo assim, 60,7\% sentiam a necessidade de aperfeiçoamento no aspecto gerencial na Saúde Coletiva e $57,1 \%$ na gestão de pessoas.

Tabela 2 - Número de profissionais que referem intenção das esferas do governo em capacitá-los para a gestão dos seus serviços

\begin{tabular}{ccc}
\hline Resposta & Frequência & Percentual \\
\hline Não & 13 & $76,47 \%$ \\
\hline Sim & 4 & $23,53 \%$ \\
\hline Total & 17 & $100 \%$ \\
\hline
\end{tabular}

Fonte: Elaborada pelas autoras.

Na Tabela 2 manifesta-se o intuito da governança em capacitar os profissionais para a gestão das suas ESFs, destacando-se que 13 sujeitos $(76,47 \%)$ relataram não ter havido intenção da alta gestão em capacitá-los para a coordenação até o momento e 4 sujeitos $(23,53 \%)$ mencionaram que houve este interesse. Tais resultados condizem com a pesquisa de Neto e Sampaio (2007), já citada anteriormente, que afirma que a maioria dos enfermeiros entrevistados em seu estudo $(96,4 \%)$ não recebeu qualificação no ato da nomeação, dificultando a compreensão do papel dos gerentes nos distintos níveis de poder dentro do Sistema de Saúde, mostrando-se possível considerar que nesse ponto reside uma das dificuldades em se concretizar os princípios e diretrizes do SUS nas ESFs.

Tabela 3 - Número de profissionais que se autoavaliam como capacitados para desenvolver as atividades gerenciais nos seus serviços

\begin{tabular}{ccc}
\hline Resposta & Frequência & Percentual \\
\hline Não & 6 & $35,29 \%$ \\
\hline Sim & 11 & $64,71 \%$ \\
\hline Total & 17 & $100 \%$ \\
\hline
\end{tabular}

Fonte: Elaborada pelas autoras.

A Tabela 3 expressa que 11 sujeitos $(64,71 \%)$ afirmaram sentir-se capacitados para atender todas as funções da administração que Ihes competem em suas ESFs, justificando-se, em sua maioria, pelo amparo teórico de sua Graduação, associado ao tempo de experiência como profissionais na APS. Estas constatações condizem com a prerrogativa de Ribeiro, Reis e Bezerra (2015), que acreditam que a capacidade e habilidades específicas para o gerenciamento são desenvolvidas durante a formação acadêmica e permanecem sendo construídas por meio das experiências diárias, durante toda a vida profissional.

Para Marquis e Huston (2002), um enfermeiro também está constantemente em desenvolvimento de suas competências gerenciais durante a sua carrei- ra. E declaram como básico que um recém-formado e recém-chegado em uma instituição de saúde deva, entre outros aspectos, utilizar a experiência dos colegas, ou seja, buscar o compartilhamento de informações para superar sua inexperiência e criar uma base sólida de poder para ir se estabelecendo como um agente influenciador, um líder.

Na Tabela 3 também está expresso que 6 entrevistados $(35,29 \%)$ relataram não se sentirem capacitados para coordenar suas equipes de forma apropriada, alegando deficiência no currículo de sua Graduação no enfoque em gestão na APS e afirmando que a maioria dos cursos dá ênfase maior à administração hospitalar. Santos et al. (2017) ressaltam em sua pesquisa, realizada em três Instituições de Ensino Superior no interior do Paraná, que os docentes participantes confirmaram que as disciplinas abordadas durante a Graduação de Enfermagem são exclusivamente assistenciais, desvinculando a prática do cuidado a do gerenciamento e dessa forma, dificultando o entendimento dos discentes em relação a sua gestão e liderança.

Na pesquisa de Almeida et al. (2014) realizada com egressos de uma universidade pública do sul do Brasil, os entrevistados demonstraram insegurança para exercer sua atividade gerencial. Este fato foi justificado pela falta de articulação entre teoria e prática, não proporcionada de forma suficiente na carga horária da disciplina de Administração em Enfermagem da grade curricular de sua Graduação, assim como carência de exercícios práticos simulando situações gerenciais, dificuldade de se colocar como "enfermeiro" durante os estágios curriculares, além da falta de interesse pelas disciplinas, envolvendo temas de gestão durante a Graduação. A partir disso, os autores sugerem a análise das disciplinas mantidas na grade curricular das Graduações em Enfermagem, haja vista a necessidade de se intensificar o estudo dos aspectos gerenciais que perpassam a práxis da Enfermagem e tornar as disciplinas gerenciais o mais teórico-práticas possível, impulsionando o discente a pensar como gerente.

Na pesquisa de Santos e Feitosa (2015) os enfermeiros entrevistados expuseram dificuldades em manter-se atualizados por conta própria, devido ao custo elevado de Pós-Graduações e participação em demais eventos. Os sujeitos pesquisados ainda argumentaram que há repetição de temas abordados em congressos voltados para a área de Enfermagem, os quais frequentemente não agregam novos conhecimentos. Tais constatações podem explicar a escassez de busca por qualificação ou serem entendidas como uma autodefesa dos sujeitos, atribuído à falta de interesse e iniciativa em se especializar e atualizar na sua área de atuação. 
Tabela 4 - Número de profissionais conforme relatam embasar o seu método de gestão

\begin{tabular}{|c|c|c|}
\hline Resposta & Frequência & Percentual \\
\hline $\begin{array}{l}\text { Processo } \\
\text { Administrativo }\end{array}$ & 8 & $47,06 \%$ \\
\hline Outros métodos & 1 & $5,88 \%$ \\
\hline Nenhum método & 8 & $47,06 \%$ \\
\hline Total & 17 & $100 \%$ \\
\hline
\end{tabular}

A Tabela 4 refere-se aos métodos de gestão que direcionam o exercício dos profissionais participantes da pesquisa. Neste quesito, 8 sujeitos $(47,06 \%)$ referiram basear-se nas funções do processo administrativo, ou seja, o ciclo de planejar, organizar, dirigir e controlar, o qual, segundo Santos et al. (2015) é o mais utilizado pelos enfermeiros na gestão do cuidado.

Os outros $47,06 \%$ afirmaram que não fazem uso de nenhuma metodologia específica para nortear suas atividades gerenciais. Trabalham com a resolução das questões conforme elas surgem, não havendo uma organização prefixada. Fato que também foi verificado na revisão de literatura de Silva (2012), que concluiu que a maioria dos enfermeiros, coordenadores das ESFs, não possuía uma estrutura gerencial estabelecida, dificultando as ações proativas na área da sua US, ora no diagnóstico de saúde da população e criação de um plano para $o$ atendimento das necessidades prévias dos indivíduos, ora no quesito de organização dos recursos humanos da própria equipe.

Também é possível correlacionar a forma de gestão "livre" dos sujeitos anteriormente citados com a Teoria de Liderança Situacional e Contingencial, caracterizada por Marquis e Huston (2002) pela sua versatilidade de estilo de liderança conforme as situações. Essa teoria sugere que nenhuma espécie de liderança é suficiente para todas as situações, suscitando a ideia de que um enfermeiro gestor e líder necessita de várias combinações de comportamento de gestão e liderança, conforme a natureza da circunstância, sua capacitação e habilidade dos subordinados.

Ainda entre os sujeitos, 1 (5,88\%) relatou que faz uso do método de gestão participativa, mencionando não ter o intuito de ser o gerente principal de sua ESF, pois não se considera capacitado para coordenar a Estratégia como um todo, argumentando que sua Graduação não o preparou para tal função e encontrou nesse método a equiparação do poder e tomada de decisão entre todos os membros da equipe.

Carvalho et al. (2016) expõem a gestão participativa como um método gerencial modelo para o cumprimento dos princípios do SUS, principalmente no âmbito da participação social, pois objetiva garantir que os profissionais e usuários contribuam com o desenvolvimento do sistema de saúde do país e, também, se corresponsabilizem pela sua saúde. Seguem destacando, porém, que, mesmo nesse método, não se exclui a presença de um profissional para coordenar os serviços. Pelo contrário, os autores destacam como desvantagem o despreparo para o exercício dessa função, visível em muitos locais que prestam cuidados em saúde, e apontam como dificuldade para o estabelecimento da gestão participativa a má administração.

De modo geral, entre os entrevistados, há um grande número de profissionais que não têm uma estrutura gerencial estabelecida ou não querem ser vistos como coordenadores, o que pode ser percebido como um inconveniente para colocar em prática a nova linha de cuidado preconizada pelo SUS, que enfatiza ações para prevenção de doenças e agravos. E consequentemente, o retardo dessas atividades de vigilância em saúde dificulta a compreensão e adaptação da população à nova orientação de saúde que o SUS antepõe.

Santos et al. (2015) consideram indispensável para os enfermeiros conhecerem as teorias gerais de Administração para nortear o gerenciamento do cuidado e de políticas públicas com clareza e segurança. Silva (2012) corrobora a afirmação dos autores anteriores quando descreve que não se deve ter uma estrutura gerencial engessada e inflexível, pois ela afeta a autonomia e proatividade dos profissionais, mas que uma base norteadora de ações é imprescindível para a eficiência e eficácia dos serviços nas ESFs.

Tabela 5 - Número de profissionais conforme seu conhecimento sobre os níveis de planejamento na saúde do município e nas suas ESFs

\begin{tabular}{|c|c|c|}
\hline Resposta & Frequência & Percentual \\
\hline \multicolumn{3}{|c|}{ Planejamento Estratégico } \\
\hline Sim & 5 & $29,41 \%$ \\
\hline Não & 12 & $70,59 \%$ \\
\hline Total & 17 & $100 \%$ \\
\hline \multicolumn{3}{|c|}{ Planejamento Tático } \\
\hline Sim & 4 & $23,53 \%$ \\
\hline $\begin{array}{l}\text { Parcialmente } \\
\text { (Grupos) }\end{array}$ & 9 & $52,94 \%$ \\
\hline Não & 4 & $23,53 \%$ \\
\hline Total & 17 & $100 \%$ \\
\hline \multicolumn{3}{|c|}{ Planejamento Operacional } \\
\hline Sim & 17 & $100 \%$ \\
\hline Total & 17 & $100 \%$ \\
\hline
\end{tabular}

Fonte: Elaborada pelas autoras. 
Na Tabela 5 é possível observar que há predominância do desconhecimento sobre o planejamento estratégico das Secretarias Municipais de Saúde (SMS), haja vista que 12 sujeitos $(70,59 \%)$ referiram que não o conhecem, mesmo este sendo preconizado como obrigatório pela Portaria no 2.135 de 2013. Esta Portaria estabelece a criação de um Plano de Saúde válido por 4 anos, que defina objetivos, diretrizes e metas a serem alcançados com base na necessidade da população local e consequentemente, devendo nortear o trabalho dos profissionais nas ESFs durante esse período (BRASIL, 2013).

Na pesquisa realizada por Saliba et al. (2013) em 3 municípios do Estado de São Paulo verificou-se que os Planos Municipais de Saúde (PMS) estavam com seus períodos de vigência vencidos, porém ainda eram válidos devido à ausência de outros para substituí-los. Também foi possível observar que ocorreram deficiências na elaboração do Plano quanto aos seus subsídios, definição de objetivos de forma estruturada e direta, exposição da distribuição de recursos, além da falta de descrição da participação e controle social, por meio das Conferências Municipais de Saúde e Conselho Municipal de Saúde. Os autores concluíram que nos municípios estudados não foi dada a devida atenção ao PMS e que seria necessário capacitar os gestores, por meio de educação permanente, quanto à importância da construção sistemática do PMS, a divulgação do resultado final para os colaboradores da SMS e comunidade, e a utilização do Plano para orientar o trabalho a ser desenvolvido.

O PMS é flexível e precisa ser adaptado conforme as necessidades da população, que são dinâmicas, e recursos materiais disponíveis, por isto tem-se a Programação Anual de Saúde (PAS), ou seja, o planejamento tático-operacional da SMS, em que é realizada a análise do PMS e escolhido o que será operacionalizado no ano específico, metas e alocação de recursos financeiros. Na tabela anterior observa-se que 4 sujeitos $(23,53 \%)$ relataram conhecer o planejamento tático de sua SMS; 9 (52,94\%) afirmaram que conhecem parcialmente, enfocando na exigência da PAS em se criar grupos de saúde nas microáreas e 4 sujeitos $(23,53 \%)$ disseram não ter conhecimento algum sobre a PAS em seu município. Situações que podem representar um fator preocupante para a saúde local, pois, sendo estas informações as que orientam as atividades diárias, deveriam ser conhecidas por todos os envolvidos no processo.

No estudo de Saliba et al. (2013) também foi constatado que houve negligência na PAS, pois nenhum dos três municípios participantes da pesquisa a realizaram. Diferente da situação expressa por Cruz (2014), que analisou a relação do planejamento e o Relatório Anual de Gestão (RAG) em um município na Bahia, constatando que o PMS, as Programações Anuais de Saúde e o RAG foram realizados com sucesso, auxiliando na tomada de decisão dos profissionais e melhorando a qualidade do atendimento oferecido à população.

Ainda na Tabela 5 está expresso que os 17 profissionais entrevistados (100\%) possuem planejamento operacional para orientar as suas atividades diárias, contando com rotinas, normas e protocolos que thes dão maior autonomia nas tomadas de decisão no nível operacional e contribuem para os colaboradores da instituição agirem de forma padronizada.

Tabela 6 - Número de profissionais conforme relato de motivação da sua equipe

\begin{tabular}{ccc}
\hline Resposta & Frequência & Percentual \\
\hline Não & 5 & $29,41 \%$ \\
\hline Sim & 12 & $70,59 \%$ \\
\hline Total & 17 & $100 \%$ \\
\hline
\end{tabular}

Fonte: Elaborada pelas autoras.

A Tabela 6 expõe que 12 sujeitos (70,59\%) descrevem sua equipe como motivada para exercer sua função, enquanto $5(29,41 \%)$ afirmam que não, devido à falta da divulgação dos resultados e reconhecimento pelo trabalho realizado há tantos anos.

A motivação passou a ser estudada na década de 20 do século 20, durante a Revolução Industrial, pois nesse momento muitos trabalhadores começaram a manifestar insatisfação com sua atividade profissional. Este fato gerou interesse de cientistas da Administração em analisar as causas da insatisfação e a relação entre satisfação do trabalhador e nível de produção. Após esse período foram propostas teorias envolvendo as relações humanas, dando maior importância ao elemento humano, sugerindo as tomadas de decisão participativas para incentivar no trabalhador o sentimento de pertencimento à organização e concluindo que um profissional realizado com suas atividades exerce seu trabalho com empenho e busca atingir os objetivos organizacionais (MARQUIS; HUSTON, 2002).

Evangelista e Costa (2008) afirmam que as pessoas são motivadas de diversas formas no mercado de trabalho, seja pelo reconhecimento de suas necessidades humanas, ou ainda pelo progresso dos benefícios relativos ao cargo. Salientam que se deve considerar que as pessoas reagem de diferentes maneiras conforme a situação a que estão expostas, devido as 
suas habilidades, capacidades, conhecimentos, personalidade, expectativas, objetivos pessoais e histórias particulares.

Como fatores desmotivadores nos serviços públicos cita-se, principalmente, a burocracia, "[...] uma vez que um único processo, na maioria das vezes, demora dias a ser resolvido e sempre depende de outras pessoas para ser realizado" (EVANGELISTA; COSTA, 2008 , p. 2). São citados ainda outros fatores, como a falta de infraestrutura, recursos humanos e materiais, podendo levar os profissionais, em muitos momentos, à impossibilidade de rearranjarem o trabalho e se sentirem impotentes. Consequentemente, Dejours (1993) defende que ocorrerá sofrimento, sentimento de desprazer e tensão em relação as suas atividades e, se este acúmulo persistir, a capacidade de conter seus sentimentos cessa e eles transbordam, podendo desencadear perturbações somáticas (apud KATSURAYAMA; PARENTE; MORETTI-PIRES, 2016).

Dessa forma, pode ser considerado que a desmotivação instiga a insatisfação com as suas atividades e gera atitudes de negação e resistência, enquanto a motivação leva à satisfação e atitudes de superação e improvisação positivas, mostrando-se fundamental que os gestores estejam sempre atentos para despertar a motivação na sua equipe.

$\mathrm{Na}$ atual pesquisa a motivação da equipe mostrou-se, do ponto de vista dos entrevistados, satisfatória, fato que também ocorreu no estudo de Evangelista e Costa (2008), em que $68,37 \%$ dos servidores públicos entrevistados sempre estiveram felizes com as suas atividades diárias. Tal evidência corrobora o estudo de Oliveira e Silva (2012), em que a maioria dos sujeitos participantes (49\%), funcionários da saúde pública municipal, encontravam-se satisfeitos com o seu trabalho.

Tabela 7 - Número de profissionais que referem utilizar indicadores como instrumentos de controle de desempenho

\begin{tabular}{ccc}
\hline Resposta & Frequência & Percentual \\
\hline Não & 9 & $52,94 \%$ \\
\hline Sim & 8 & $47,06 \%$ \\
\hline Total & 17 & $100,00 \%$ \\
\hline \multicolumn{3}{c}{ Fonte: Elaborada pelas autoras. }
\end{tabular}

Os indicadores são instrumentos de controle de desempenho organizacional e são essenciais para a avaliação dos resultados dos serviços realizados e indispensáveis para a correção e criação de novos objetivos e metas (MENEZES; INNOCENZO, 2013). Reconhecendo sua importância para o adequado funciona- mento da coordenação, aponta-se como significativo o grande número de profissionais que não os utilizam nos municípios estudados, 52,94\%, podendo comprometer a eficácia da análise dos resultados alcançados nos serviços realizados. Também cabe ressaltar que 8 sujeitos $(47,06 \%)$ responderam que fazem uso dos indicadores como instrumentos de controle porque são estipulados pelo Programa de Melhoria do Acesso e da Qualidade (PMAQ), subentendendo-se que utilizam esta ferramenta devido à imposição do Programa.

Resultado semelhante encontra-se na pesquisa de Paes et al. (2015) sobre o uso de indicadores como ferramenta de gestão na ESF em um município de médio porte do Estado de Santa Catarina, pois revelaram que os enfermeiros gerentes reconhecem a importância do instrumento para o processo de gerenciamento na APS, contudo evidenciaram "[...] certo distanciamento entre os conceitos apresentados pelos profissionais e aquilo que relatam utilizar na prática" (p. 47).

Além de que, os autores concluíram que os indicadores utilizados pelos enfermeiros são predeterminados pela SMS ou Ministério da Saúde, havendo poucos relatos de novas construções, no sentido de estabelecer indicadores apropriados à realidade local de sua área de atuação.

Tabela 8 - Número de profissionais que relatam fazer o controle das atividades desempenhadas pela sua equipe por meio de relatórios

\begin{tabular}{ccc}
\hline Resposta & $\begin{array}{c}\text { Frequência } \\
\text { Presença de relatórios }\end{array}$ & Percentual \\
\hline Não & 2 & $11,76 \%$ \\
\hline Sim & $\mathbf{1 5}$ & $\mathbf{8 8 , 2 4 \%}$ \\
\hline Total & 17 & $100,00 \%$ \\
\hline \multicolumn{3}{|c}{ Análise dos relatórios } \\
\hline Não & 12 & $80,00 \%$ \\
\hline Sim & 3 & $20,00 \%$ \\
\hline Total & $\mathbf{1 5}$ & $\mathbf{1 0 0 , 0 0 \%}$ \\
\hline
\end{tabular}

Fonte: Elaborada pelas autoras.

A Tabela 8 denota que a maioria dos sujeitos pesquisados, 15 (88,24\%), tem elaborado nas suas ESFs relatórios sobre as atividades desempenhadas pela equipe. Circunstância necessária para alimentação mensal e sistemática do Banco de Dados Nacional do Sistema de Informação em Saúde para a Atenção Básica (Sisab), conforme a Portaria no 3.462. Desses 15 profissionais que têm relatórios disponíveis, porém, apenas $3(17,65 \%)$ efetuam a análise desses documentos. Essa situação compromete a verificação da eficácia de ações e estratégias, assim como a avaliação do alcance de objetivos e metas pactuadas, gerando 
apenas um banco de dados isolados, sem informações fundamentadas capazes de suscitar conhecimento e embasar tomadas de decisão conscientes (OHIRA; JUNIOR; NUNES, 2014).

Ohira, Junior e Nunes (2014), em sua pesquisa sobre práticas gerenciais na APS em 40 municípios de pequeno porte do norte do Paraná, verificaram que apenas $64,5 \%$ dos gerentes discutiam as metas e indicadores com sua equipe e que $61,1 \%$ realizavam avaliação das ações, serviços de saúde e trabalhadores, demonstrando insuficiência no controle de desempenho organizacional nos municípios estudados.

Este cenário também poder ser subentendido na pesquisa de Paes et al. (2015), já mencionada anteriormente, em que se citou o desinteresse da maioria dos enfermeiros gestores em criar novos indicadores voltados ao atendimento das necessidades cotidianas de sua população, ao invés de utilizar os padronizados pela sua SMS e pelo MS. Isto sugere uma análise e controle superficiais do perfil de saúde de sua população e da repercussão das atividades realizadas em sua ESF.

\section{CONCLUSÃO}

Com o presente estudo foi identificado que a maioria dos enfermeiros não possui capacitação específica na área de gerenciamento e que as esferas do governo, envolvidas com a gestão dos serviços de saúde, não têm considerado o aperfeiçoamento dos coordenadores das ESFs. Assim como, por mais que grande parte dos profissionais sinta-se apta para a gestão, suas respostas não condizem com uma atuação hábil nessa área, em virtude da fragilidade no conhecimento do PMS e da PAS, os quais deveriam orientar a conduta das suas equipes diariamente; e a inobservância dos relatórios e indicadores de saúde, comprometendo a verificação da eficiência e eficácia de ações e estratégias e a então possível adaptação de objetivos, metas e intervenções locais.

Em síntese, por meio da análise dos dados da pesquisa, verificou-se que nem o Processo Administrativo Neoclássico, nem outros métodos de gestão estão consolidados nas ESFs. A execução da coordenação dos enfermeiros ainda é deveras baseada na improvisação e empirismo. Tal realidade pode retardar o desenvolvimento da melhoria dos serviços do SUS, necessitando de novas pesquisas nesse campo, capazes de tencionar o debate sobre a importância da qualificação dos processos de gerenciamento na ESF e a revisão dos currículos do curso de Bacharelado em
Enfermagem, a fim de avaliar se ocorre uma predominância do assistencialismo e administração hospitalar em detrimento da gestão na APS.

\section{REFERÊNCIAS}

ALBUQUeRQUe, M. I. N. Uma revisão sobre as Políticas Públicas de Saúde no Brasil. 2015. Disponível em: https://ares. unasus.gov.br/acervo/handle/ARES/3333. Acesso em: 18 ago. 2017.

ALMEIDA, J. Habilidades e competências do enfermeiro no gerenciamento dos serviços na Atenção Primária à Saúde. Bom Despacho - MG. 2014. Trabalho (Conclusão de Curso) - UFMG, 2014.

ALMEIDA, M. L. et al. Egressos de uma universidade pública e perspectivas de atuação no gerenciamento em enfermagem. Revista Rene, v. 15, n. 6, p. 933-941, nov./dez. 2014.

ALVES, V. L. S. Gestão da qualidade: ferramentas utilizadas no contexto contemporâneo de saúde. 2. ed. São Paulo: Martinari, 2012.

BARBOSA, A. R. A. F. A importância do conhecimento em administração para a prática profissional do corpo de Enfermagem do Programa Saúde da Família do Distrito Sanitário V - Campina Grande - PB. 2014. Disponível em: http://dspace.bc.uepb.edu.br/jspui/bitstream/123456789/4708/1/ PDF\%20-\%20Alexandrini\%20Romanowski\%20Androukovitch\%20Felix\%20Barbosa.pdf. Acesso em: 10 jun. 2016.

BRASIL, M. S. Portaria no 2.436: Aprova a Política Nacional de Atenção Básica, estabelecendo a revisão de diretrizes para a organização da Atenção Básica, no âmbito do Sistema Único de Saúde (SUS). 2017. Disponível em: http://bvsms.saude.gov.br/bvs/saudelegis/gm/2017/ prt2436_22_09_2017.html. Acesso em: 15 jul. 2017.

BRASIL, M. S. Portaria no 2.135: Estabelece diretrizes para o processo de planejamento no âmbito do Sistema Único de Saúde (SUS). 2013. Disponível em: http://bvsms.saude.gov. br/bvs/saudelegis/gm/2013/prt2135_25_09_2013.html. Acesso em: 18 ago. 2017.

CARVALHO, J. B. L. et al. Gestão participativa e a interface com trabalhadores do Sistema Único de Saúde: uma revisão integrativa. Revista Brasileira da Educação Profissional e Tecnológica, v. 2, n. 11, p. 131-139, 2016.

CHIAVENATO, I. Teoria geral da administração. 6. ed. Rio de Janeiro: Elsevier. 2001.

CRUZ, J. S. et al. Gestão da Atenção Básica no Sistema Único de Saúde de Santo Antônio de Jesus, Bahia, 2009-2012. Revista Baiana de Saúde Pública, v. 38, n. 1, p. 49-66, jan./ mar. 2014.

DIAS, C. L. C. et al. Enfermeiro como gerenciador da Rede Básica de Saúde. Trabalho Completo apresentado no Sepe. Santa Maria: Centro Universitário Franciscano, 2012.

EVANGELISTA, W. L.; COSTA, F. R. Motivação de funcionários do serviço público federal de Bambuí-MG. 2008. Disponível em: http://www.bambui.ifmg.edu.br/jornada_cientifica/ str/artigos_aprovados/administracao/90-PT-3.pdf. Acesso em: 27 ago. 2017. 
IBGE. Instituto Brasileiro de Geografia e Estatística. Censo Demográfico. 2010. Disponível em: http://www.cidades. ibge.gov.br/xtras/home.php. Acesso em: 20 maio 2016.

KATSURAYAMA, M.; PARENTE, R. C. P.; MORETTI-PIRES, R. O. O trabalhador no programa saúde da família no interior do Estado do Amazonas: um estudo qualitativo. Trab. Educ. Saúde, v. 14, n. 1, p. 183-198, jan./abr. 2016.

MACHADO, M. H. et al. Características gerais da enfermagem: o perfil sociodemográfico. Enfermagem em Foco, v. 7, p. 35-62. 2016.

MARQUIS, B. L.; HUSTON, C. J. Administração e liderança em enfermagem. 2. ed. Porto Alegre: Artmed. 2002.

MENEZES, P. I. F. B.; INNOCENZO, M. D'. Dificuldades vivenciadas pelo enfermeiro na utilização de indicadores de processo. Revista Brasileira de Enfermagem, v. 66, n. 4, p. 571577, jul./ago. 2013.

NETO, F. R. G. X.; SAMPAIO, J. J. C. Gerentes do território na Estratégia Saúde da Família: análise e perfil de necessidades de qualificação. Revista Brasileira de Enfermagem, v. 60, n. 6, p. 687-695, nov./dez. 2007.

OHIRA, R. H. F.; JUNIOR, L. C.; NUNES, E. F. P. A. Perfil dos gerentes de Atenção Primária à Saúde de municípios de pequeno porte do norte do Paraná, Brasil. Revista Ciências e Saúde Coletiva, v. 19, n. 2, p. 393-400, 2014.

OLIVEIRA, T. L.; SILVA, L. C. J. Motivação dos servidores públicos: um caso na saúde pública na Amazônia. Revista de Administração de Roraima, v. 2, n. 1, p. 178-195. 2012.

PAES, L. G. et al. O uso de indicadores como ferramenta de gestão na Estratégia Saúde da Família. Revista de Enfermagem da UFSM, v. 5, n. 1, p. 40-49, jan./mar. 2015.
POÇAS, K. C.; FREITAS, L. R. S.; DUARTE, E. C. Censo de estrutura da Atenção Primária à Saúde no Brasil (2012): estimativas de coberturas potenciais. Epidemiologia e Serviços de Saúde, v. 26, n. 2, p. 275-284, abr./jun. 2017.

RIBEIRO, M.; SANTOS, S. L.; MEIRA, T. G. B. M. Refletindo sobre liderança em Enfermagem. Escola Anna Nery, v. 10, n. 1, p. 109-115. 2006.

RIBEIRO, A. B. A.; REIS, R. P.; BEZERRA, D. G. Gestão em Saúde Pública: um enfoque no papel do Enfermeiro. Revista Brasileira de Ciências da Saúde, v. 19, n. 3, p. 247-252, 2015.

SALIBA, N. A. et al. Plano Municipal de Saúde: análise do instrumento de gestão. Bioscience Journal, v. 29, n. 1, p. 224-230, jan./fev. 2013.

SANTOS, P. R. et al. Ensino do gerenciamento e suas implicações à formação do enfermeiro: perspectivas de docentes. Ciência, Cuidado e Saúde, v. 16, n. 1, p. 1-8, jan./mar. 2017.

SANTOS, J. C.; FEITOSA, M. G. G. Gestão em saúde: as dificuldades intrapessoais do enfermeiro no exercício profissional. Journal of Nursing, v. 9, n. 2, p. 790-798, fev. 2015.

SANTOS, A. F. et al. Percepção dos enfermeiros quanto a gerência do cuidado em uma unidade básica de saúde da família em Maracanaú-CE. Revista Diálogos Acadêmicos, v. 4, n. 1, p. 53-58, jan./jun. 2015.

SILVA, F. H. C. A atuação dos enfermeiros como gestores em Unidades Básicas de Saúde. Revista de Gestão em Sistemas de Saúde, v. 1, n. 1, p. 67-82, jan./jun. 2012.

SILVA, V. G.; MOTTA, M. C. S.; ZEITOUNE, R. C. G. A prática do enfermeiro na Estratégia Saúde da Família: o caso do município de Vitória/ES. Revista Eletrônica de Enfermagem, v. 12, n. 3, p. 441-448. 2010. 